\title{
COVID-19-Induced Pancytopenia in a Major Molecular Response CML Patient on Dasatinib: A Case Report and Review of the Literature
}

\author{
Balraj Singh ${ }^{1}$, Sarah Ayad ${ }^{1}$, Parminder Kaur ${ }^{2}$, Vinod Kumar ${ }^{1}$, Sachin Gupta ${ }^{3}$, Michael Maroules ${ }^{1}$ \\ ${ }^{1}$ Department of Hematology \& Oncology, Saint Joseph's University Medical Center, Paterson, NJ, USA \\ ${ }^{2}$ Department of Cardiology, Saint Joseph's University Medical Center, Paterson, NJ, USA \\ ${ }^{3}$ Department of Internal Medicine, Tower Health Reading Hospital, West Reading, PA, USA
}

\section{Doi: 10.12890/2021_002233 - European Journal of Case Reports in Internal Medicine - @ EFIM 2020}

Received: 20/12/2020

Accepted: $23 / 12 / 2020$

Published: $12 / 01 / 2021$

\begin{abstract}
How to cite this article: Singh B, Ayad S, Kaur P, Kumar V, Gupta S, Maroules M. COVID-19-induced pancytopenia in a major molecular response cml patient on dasatinib: a case report and review of the literature. EJCRIM 2021;8: doi:10.12890/2020_002233.
\end{abstract}

Conflicts of Interests: The Authors declare that there are no competing interests.

This article is licensed under a Commons Attribution Non-Commercial 4.0 License

\section{ABSTRACT}

Severe acute respiratory syndrome coronavirus 2 (SARS-CoV-2), the virus that causes coronavirus disease 2019 (COVID-19), has caused a global health crisis. COVID-19 can have a multifaceted presentation, and a wide range of complications and outcomes may emerge based on the severity and comorbidities of the infected patient. We report the case of a 42-year-old man with a history of chronic myeloid leukaemia (CML) on dasatinib (in major molecular response) who was diagnosed with COVID-19 and developed pancytopenia. Our case report and review of available publications add to the limited literature available regarding COVID-19 in CML.

\section{LEARNING POINTS}

- Our case report and review of the literature highlight the multifaceted response in chronic myeloid leukaemia (CML) patients to COVID-19 (respiratory symptoms, pancytopenia, severe haemolytic anaemia and haemophagocytic lymphohistiocytosis, disseminated erythematous papular skin rash).

- The rates of intensive care unit admission and mechanical ventilation support, and lengths of hospital stay were lower in COVID-19 patients with CML receiving tyrosine kinase inhibitors (TKI) compared with an age, gender and comorbidity-matched control group.

- Our case report and review of the literature suggest a possible protective effect of TKI therapy against COVID-19 in patients with CML.

\section{KEYWORDS}

Tyrosine kinase inhibitors, COVID-19, SARS-CoV-2, chronic myeloid leukemia, imatinib, dasatinib, nilotinib, cancer

\section{INTRODUCTION}

In December 2019, novel severe acute respiratory syndrome coronavirus 2 (SARS-CoV-2), which causes coronavirus disease 19 (COVID-19), emerged in Wuhan, Hubei province, China and has since caused significant health, psychological and economic problems worldwide. In over a year, more than 72 million confirmed cases and more than 1.6 million deaths have been reported globally according to the World Health Organization, a significant number that reflects the magnitude of the pandemic and the current worldwide situation. The clinical manifestations of SARS-CoV-2 vary from mild self-limiting flu-like symptoms such as fever, chills, cough, shortness of breath, fatigue and body aches, to severe respiratory failure, renal, neurological, gastrointestinal and haematological involvement and eventually multi-organ failure ${ }^{[1]}$.

The main effects of the virus are attributed to a heightened inflammatory response and hyperactivation of T cells and increased production of inflammatory cytokines including interleukins (IL-6, IL-1), tumour necrosis factor- $\alpha$ (TNF- $\alpha$ ) and interferon- $y$ (INF- $\gamma$ ), which triggers an 
inflammatory cascade that leads to plasma leakage, vascular permeability and pulmonary damage ${ }^{[2]}$. Although extrapulmonary effects of COVID-19 have been documented, the implications for immunocompromised patients such as those with cancer are not fully known. In this article, we report the case of a 42-year-old man with a history of chronic myeloid leukaemia (CML) on dasatinib (in major molecular response) who was diagnosed with COVID-19 and developed pancytopenia, and describe a review of the literature on the outcome of COVID-19 in patients on tyrosine kinase therapy.

\section{CASE DESCRIPTION}

We report the case of a 42-year-old man with no significant comorbidities who was diagnosed with CML at the age of 28 and placed on imatinib. However, he was non-compliant with medication and was lost to follow-up. In February 2020, the patient presented to the emergency department (ED) with a 2-month history of left upper quadrant pain associated with a 20 pound weight loss. Physical examination was remarkable for massive splenomegaly. At that time, the patient was found to have an elevated white blood count (WBC) of $330.3 \times 10^{3} /$ $\mathrm{mm}^{3}$ (4.5-11.0), haemoglobin (Hgb) $7.3 \mathrm{~g} / \mathrm{dl}$ (13.5-17.5) and platelets $243 \mathrm{~K} / \mathrm{mm}^{3}$ (140-440). The differential showed basophils $4 \%(0-2)$, blasts $10 \%(0)$, promyelocytes 6\% (0) and LDH 700 units/l (140-271). Flow cytometry of peripheral blood showed 6\% myeloblasts and 8\% basophils, and peripheral blood RT-PCR BCR-ABL showed e13a2 (p210-b3a2) 75\%, e14a2 (p210-b2a2) 25\%, and e1a2 (p190) 0.03\%. Bone marrow aspirate flow cytometry showed $6.5 \%$ myeloblasts and $9.4 \%$ basophils. Bone marrow biopsy showed markedly hypercellular marrow, the presence of increased micromegakaryocytes in large aggregates and markedly increased reticulin fibrosis. The patient was diagnosed with chronic phase CML and was started on dasatinib on 14 February 2020. He continued to follow-up and was compliant with dasatinib. In March 2020, the WBC decreased to $16.1 \times 10^{3} / \mathrm{mm}^{3}$ and in April 2020 the patient achieved a complete haematological response (WBC $8.43 \times 10^{3} / \mathrm{mm}^{3}$, Hgb $12.6 \mathrm{~g} / \mathrm{dl}$, platelets $163 \mathrm{~K} / \mathrm{mm}^{3}$ ). In October 2020, the patient achieved major molecular response (peripheral blood RT-PCR BCR-ABL <0.1\%).

In December 2020, the patient presented to the ED with a 1-week history of unremitting fevers despite Tylenol use and chills after exposure to a friend who had 'flu-like symptoms'. The patient's spouse also had upper respiratory symptoms around the same time. He denied shortness of breath, cough, abdominal pain, nausea, vomiting, diarrhoea, new rashes, dysuria or myalgias. In the ED, initial vital signs were significant for: temperature $37.1^{\circ} \mathrm{C}$, heart rate $98 \mathrm{bpm}$, respiratory rate 18 , blood pressure $121 / 61 \mathrm{mmHg}$, and SpO2 96-98\% on room air at rest. Laboratory test results were significant for WBC $4.4 \times 10^{3} / \mathrm{mm}^{3}$, Hgb $11.5 \mathrm{~g} / \mathrm{dl}$, platelets $106 \mathrm{~K} / \mathrm{mm}^{3}$, and elevated inflammatory markers consistent with COVID pneumonia including ferritin $355.0 \mathrm{ng} / \mathrm{ml}$ (16.4-294.0), CRP $67.5 \mathrm{mg} / \mathrm{l}$ ( $\leq 9.9)$, LDH 303 units/l (140-271), D-dimer $1.42 \mu \mathrm{g} / \mathrm{ml}(\leq 0.50)$ and ESR $78 \mathrm{~mm} / \mathrm{hr}(<10)$. A chest x-ray showed bilateral patchy infiltrates (Fig. 1). A computed tomography (CT) angiogram of the chest showed bilateral infiltrates in both lungs (Fig. 2).

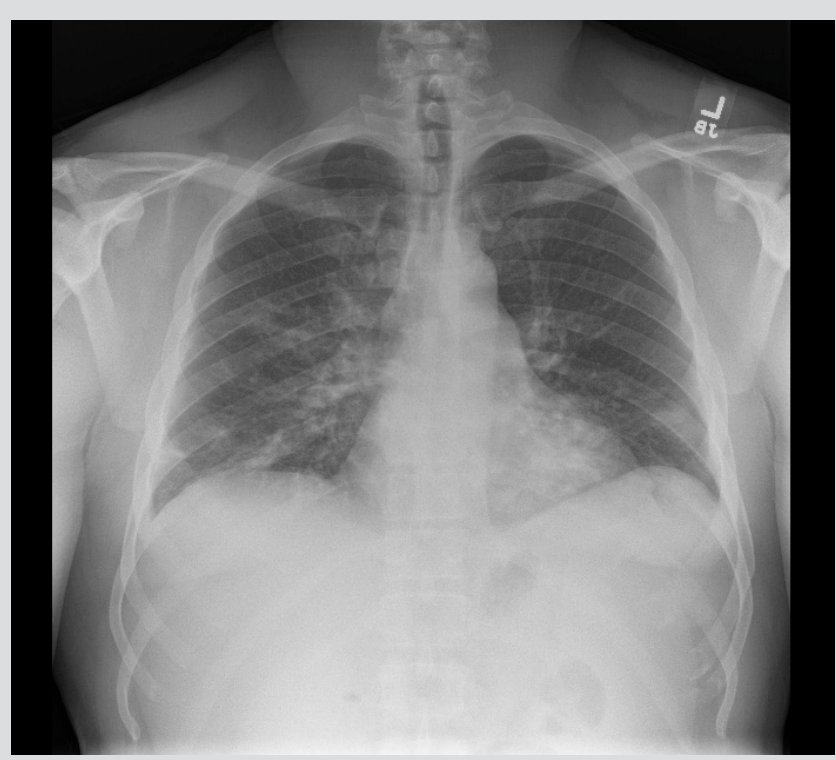

Figure 1. Chest $x$-ray showing patchy bilateral pulmonary infiltrates

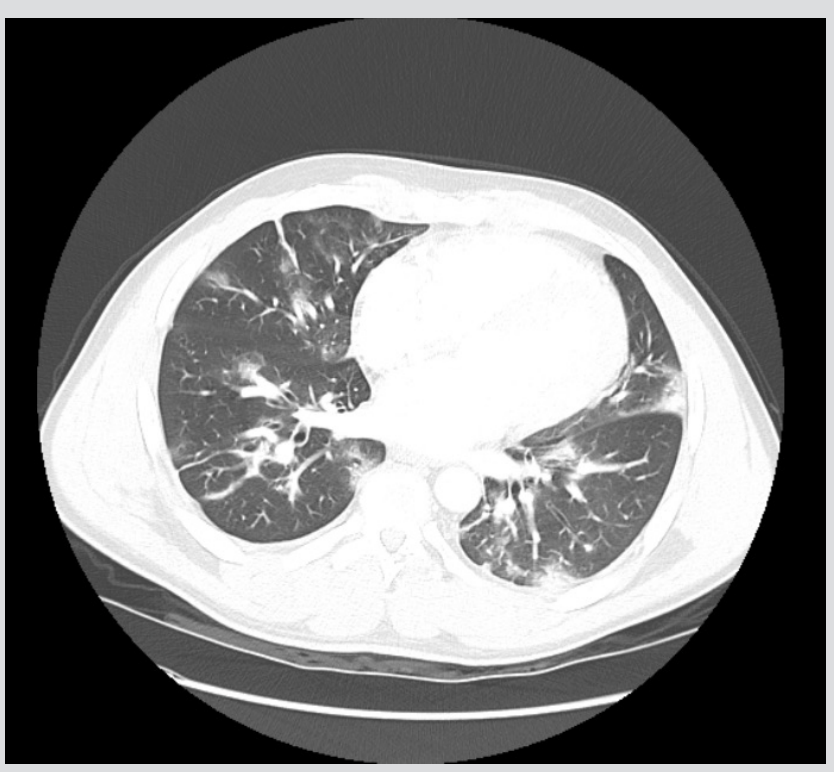

Figure 2. Computed tomography (CT) angiogram of the chest showed patchy infiltrates in both lungs 
A nasopharyngeal swab tested positive for SARS-CoV-2 by real-time reverse-transcription polymerase chain reaction ( $r$ RT-PCR) assay. The patient was haemodynamically and clinically stable and was discharged home from the ED with instructions to continue dasatinib for CML and continue follow-up with the haematology oncology clinic. The patient was re-evaluated 2 weeks after presentation via telehealth; his respiratory symptoms had resolved and blood work showed improving counts, with WBC $6.9 \times 10^{3} / \mathrm{mm}^{3}$, Hgb $13.4 \mathrm{~g} / \mathrm{dl}$, and platelets 124 $\mathrm{K} / \mathrm{mm}^{3}$.

\section{DISCUSSION}

CML is a myeloproliferative neoplasm that consists mainly of proliferating granulocytes that are known to have the Philadelphia chromosome translocation $\mathrm{t}(9 ; 22)$. CML can be classified into three different phases: the chronic phase, accelerated phase and blast phase ${ }^{[3]}$.

A nationwide analysis conducted in China by Liang et al. in March 2020 concluded that cancer patients might have a high risk of COVID-19 and had a poor outcome compared with those without cancer. Furthermore, the authors recommended considering postponing adjuvant chemotherapy in patients who are stable ${ }^{[4]}$. In this review article, we aim to explore the prevalence and outcome of COVID-19 among patients with CML and more specifically, patients on $\mathrm{TKI}$, with the hope of guiding clinicians and patients.

We conducted a literature review by searching PubMed using the search terms 'COVID-19 and CML' and 'COVID-19 and chronic myeloid leukaemia'. This search generated eight articles and relevant information was abstracted from these studies. The publications included case reports, case series, survey questionnaires, and letters to the editor.

Abruzzese et al. reported the first case of COVID-19 in CML: a young female patient with CML on dasatinib (in deep molecular response) who was diagnosed with COVID-19 after presenting with fever. She was admitted to hospital and given antibiotics (amoxicillin and clavulanic acid) for 7 days with paracetamol as needed. This patient continued treatment with dasatinib during hospitalization and was subsequently discharged ${ }^{[5]}$. Sorà et al. reported another case of a 55-year male patient with CML (in major molecular response) on imatinib $400 \mathrm{mg}$ who was diagnosed with COVID-19 complicated by severe haemolytic anaemia and haemophagocytic lymphohistiocytosis. The patient received antiviral therapy, steroids and intravenous immunoglobulins (Ig). Haemolysis resolved and ferritin dramatically decreased after administration of Ig and full recovery was achieved after viral infection resolution ${ }^{[2]}$. It was not reported whether this patient was continued on imatinib or not. Lagziel et al. described a 58-year-old woman with a history of CML managed for 4 years with imatinib, morbid obesity, hypertension, gout and chronic kidney disease who was admitted initially for fever and respiratory distress, later complicated by a disseminated erythematous and papular skin rash; biopsy was consistent with bullous interface dermatitis ${ }^{[6]}$.

Using a questionnaire, Li et al. studied 530 subjects with CML in Hubei province during the COVID-19 pandemic. Five developed confirmed $(n=4)$ or probable $(n=1)$ COVID-19. The prevalence of COVID-19 in CML patients was 0.9\%, nine-fold higher than the $0.1 \%$ reported in normal subjects but lower than the $10 \%$ reported in hospitalized patients with other haematological cancers or the $7 \%$ in normal healthcare providers. Co-variates associated with an increased risk of developing COVID-19 among patients with CML were exposure to someone infected with SARS-CoV-2 ( $p=0.037)$, no complete haematological response $(p=0.003)$ and comorbidity ( $p=0.024)$. There was also an increased risk of developing COVID-19 in subjects with advanced phase CML $(p=0.004)$, even when they had achieved a complete cytogenetic response or major molecular response at the time of exposure to SARS-CoV-2 ${ }^{[7]}$.

Ector et al. conducted a prospective Dutch observational cohort study using survey questionnaires sent to patients with CML and to their adult housemates used as a control group. The study included 123 housemates and 148 patients with CML of whom $88.4 \%$ had taken TKI therapy. At the time of article submission, only one patient had tested positive for COVID-19. This patient was a 62-year-old man with CML on imatinib for 2 years and without any comorbidities. He was admitted to a general ward and did not require ICU admission. The authors concluded that the prevalence of COVID-19 in CML patients was $0.7 \%{ }^{[8]}$.

On 6 April 2020, Breccia et al. sent an ad hoc questionnaire to 51 Campus CML centres throughout Italy and data were gathered on 6883 CML patients; only 12 cases of confirmed COVID-19 infection (0.17\%) were reported up to the middle of April ${ }^{[9]}$. BaşCI et al. describe a retrospective study from Turkey of 16 laboratory-confirmed COVID-19 patients with CML receiving TKI and 48 age, gender, and comorbid disease-matched COVID-19 patients without cancer at a 3:1 ratio, diagnosed between 11 March 2020 and 22 May 2020 . In this report, the case fatality rate was lower in CML patients (6.3\%) than in the control group (12.8\%). Additionally, the rates of intensive care unit admission and mechanical ventilation support, and lengths of hospital stay were lower in COVID-19 patients with CML receiving TKI compared with the age, gender and comorbidity-matched control group. However, these differences were not statistically significant ${ }^{[10]}$.

Management of CML mandates the use of TKI. It was reported that, during the early stages of treatment, commercially available TKI might cause toxicity, including myelosuppression and cytopenia ${ }^{[11]}$. A higher grade of myelosuppression (grade III-IV) is generally seen in the initial stage of treatment and declines dramatically with longer duration of therapy ${ }^{[12]}$. Despite these findings, neither CML nor

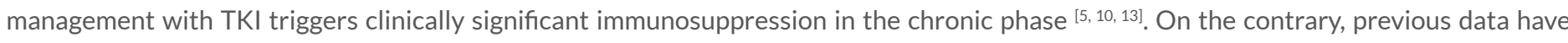
shown that TKI therapy may play a protective role against SARS-CoV-2 in vitro ${ }^{[13]}$. 
During the emergence in 2014 of Middle East respiratory syndrome coronavirus (MERS-CoV), several reports studied the possibility of antiviral drug activity against MERS-CoV and SARS-CoV. TKI including imatinib mesylate (Gleevec), nilotinib and dasatinib were among the compounds studied: imatinib and dasatinib were active against both MERS-CoV and SARS-CoV, while nilotinib inhibited SARS-CoV only ${ }^{[5,13]}$

While the exact mechanism is not known, TKI may play an important role in the replication and dissemination of the Coronaviridae through the Abelson tyrosine-protein kinase (Abl) pathway ${ }^{[13]}$. Previous data have shown that through inhibition of the Abl pathway, imatinib can halt viral replication by inhibiting viral fusion with the endosomal membrane and consequently inhibit its release in the cytoplasm and eventually its replication ${ }^{[5,9,13]}$. Others suggest that the BCR-Abl 1 tyrosine kinase may alter the host cells, rendering them 'impenetrable' by the virus by changing the actin and cytoskeletal structure and cytoskeletal function ${ }^{[14]}$. This indicates that patients with $\mathrm{PH}+$ genes are potentially less likely to be infected than healthy individuals ${ }^{[14]}$.

Moreover, previous studies have shown that imatinib can facilitate a reduction in inflammation and preserve vascular integrity. Rizzo et al. showed that imatinib decreased bronchoalveolar lavage protein, total cells, neutrophils and TNF-? release in mice models with acute lung injury and acute respiratory distress syndrome ${ }^{[15]}$. Therefore, imatinib can help reduce acute lung injury and the acute respiratory distress syndrome which is the hallmark of COVID-19 ${ }^{[10,15]}$.

\section{CONCLUSION}

While some studies argued that the prevalence of COVID-19 in patients with CML is slightly higher than in the general population, the majority of these results did not achieve statistical significance and many of the data had several limitations including selection bias and small sample size. Despite these limitations, the reported prevalence is very low at $<1 \%$, which is significant for both patients and clinicians. Many authors argue that perhaps the low prevalence reported during the pandemic may demonstrate the protective effect of TKI therapy. While the data are promising, larger prospective and perhaps randomized studies are necessary to examine the outcome of COVID-19 in patients with CML and to clarify the role of TKI therapy and its possible antiviral effect during the pandemic.

\section{REFERENCES}

1. Gupta A, Madhavan MV, Sehgal K, Nair N, Mahajan S, Sehrawat TS, et al. Extrapulmonary manifestations of COVID-19. Nat Med 2020;26(7):1017-1032.

2. Sorà F, Chiusolo P, Laurenti L, Innocenti I, Autore F, Alma E, et al. SARS CoV 2 infection in chronic myelogenous leukemia: severe hematological presentation. Transfus Apher Sci 2020;59(6):102881.

3. Eden RE, Coviello JM. Chronic myelogenous leukemia. Treasure Island (FL): StatPearls Publishing; 2020.

4. Liang W, Guan W, Chen R, Wang W, Li J, Xu K, et al. Cancer patients in SARS-CoV-2 infection: a nationwide analysis in China. Lancet Oncol 2020;21(3):335-337.

5. Abruzzese E, Luciano L, D'Agostino F, Trawinska MM, Pane F, De Fabritiis P. SARS-CoV-2 (COVID-19) and chronic myeloid leukemia (CML): a case report and review of ABL kinase involvement in viral infection. Mediterr J Hematol Infect Dis 2020;12(1):e2020031.

6. Lagziel T, Quiroga L, Ramos M, Hultman CS, Asif M. Two false negative test results in a symptomatic patient with a confirmed case of severe acute respiratory syndrome Coronavirus-2 (SARS-CoV-2) and suspected Stevens-Johnson syndrome/toxic epidermal necrolysis (SJS/TEN). Cureus 2020;12(5):e8198.

7. Li W, Wang D, Guo J, Yuan G, Yang Z, Gale RP, et al. COVID-19 in persons with chronic myeloid leukaemia. Leukemia 2020;34(7):1799-1804.

8. Ector G, Huijskens EGW, Blijlevens NMA, Westerweel PE. Prevalence of COVID-19 diagnosis in Dutch CML patients during the 2020 SARS-CoV2 pandemic. A prospective cohort study. Leukemia 2020;34(9):2533-2535.

9. Breccia M, Abruzzese E, Bocchia M, Bonifacio M, Castagnetti F, Fava C, et al. Chronic myeloid leukemia management at the time of the COVID-19 pandemic in Italy. A Campus CML survey. Leukemia 2020;34(8):2260-2261.

10. Başcı S, Ata N, Altuntaş F, Yiğenoğlu TN, Dal MS, Korkmaz S, et al. Outcome of COVID-19 in patients with chronic myeloid leukemia receiving tyrosine kinase inhibitors. J Oncol Pharm Pract 2020;26(7):1676-1682.

11. Paul TR, Uppin SG, Uppin MS, Jacob RT, Rao DR, Rajappa SJ. Evaluation of cytopenias occurring in imatinib treated chronic myeloid leukemia (CML) patients. Indian J Hematol Blood Transfus 2010;26(2):56-61.

12. Eşkazan AE. Tyrosine kinase inhibitors (TKIs) used in the management of chronic myeloid leukaemia are associated with haematologic toxicities - which TKI is the safest? Br J Clin Pharmacol 2019;85(10):2241-2243.

13. Dyall J, Coleman CM, Hart BJ, Venkataraman T, Holbrook MR, Kindrachuk J, et al. Repurposing of clinically developed drugs for treatment of Middle East respiratory syndrome coronavirus infection. Antimicrob Agents Chemother 2014;58(8):4885-4893.

14. Galimberti S, Petrini M, Baratè C, Ricci F, Balducci S, Grassi S, et al. Tyrosine kinase inhibitors play an antiviral action in patients affected by chronic myeloid leukemia: a possible model supporting their use in the fight against SARS-CoV-2. Front Oncol 2020;10(1428).

15. Rizzo AN, Sammani S, Esquinca AE, Jacobson JR, Garcia JGN, Letsiou E, et al. Imatinib attenuates inflammation and vascular leak in a clinically relevant two-hit model of acute lung injury. Am J Physiol Lung Cell Mol Physiol 2015;309(11):L1294-L304. 